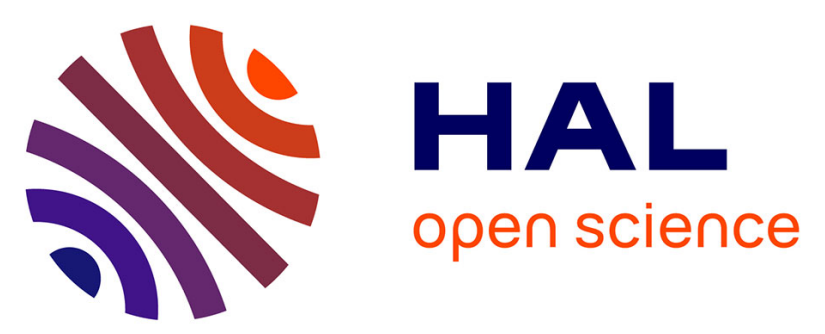

\title{
Comparison between inductive and resistive SFCL in terms of current limitation and power system transient stability
}

Gaëtan Didier, Charles-Henri Bonnard, Thierry Lubin, Jean Lévêque

\section{- To cite this version:}

Gaëtan Didier, Charles-Henri Bonnard, Thierry Lubin, Jean Lévêque. Comparison between inductive and resistive SFCL in terms of current limitation and power system transient stability. Electric Power Systems Research, 2015, 125, pp.150-158. 10.1016/j.epsr.2015.04.002 . hal-01164392

\section{HAL Id: hal-01164392 \\ https://hal.science/hal-01164392}

Submitted on 16 Jun 2015

HAL is a multi-disciplinary open access archive for the deposit and dissemination of scientific research documents, whether they are published or not. The documents may come from teaching and research institutions in France or abroad, or from public or private research centers.
L'archive ouverte pluridisciplinaire HAL, est destinée au dépôt et à la diffusion de documents scientifiques de niveau recherche, publiés ou non, émanant des établissements d'enseignement et de recherche français ou étrangers, des laboratoires publics ou privés. 


\title{
Comparison between inductive and resistive SFCL in terms of current limitation and power system transient stability
}

\author{
G. Didier*, C. H. Bonnard, T. Lubin, J. Lévêque \\ Groupe de Recherche en Electrotechnique et Electronique de Nancy, GREEN, Université de Lorraine, 54506 Vandoeuvre-lès-Nancy Cedex, \\ France.
}

\begin{abstract}
In this paper, authors present a comparative study of inductive and resistive superconducting fault current limiters (SFCL) from current limitation and power system transient stability point of view. Different types of SFCL can be used to decrease the amplitude of fault current in a power system. The two most used are resistive type (rSFCL) and inductive type (iSFCL). However, no studies have been done to show the difference between them in term of current limitation and transient stability on a same power system. We propose here to compare rSFCL and iSFCL when a fault occurs in a simple high voltage (HV) power system (IEEE 3 bus test system). To evaluate the impact of SFCL in the studied power system, time domain approach is used to evaluate the short-circuit current in the electrical power system by solving the differential equations of such circuit configuration for various SFCL impedance. For the transient stability study, authors use an approach based on the equal area criterion to evaluate the critical clearing angle (CCA) and a time domain approach to evaluate the critical clearing time (CCT) for the two types of SFCL. Results show that the two SFCLs have different impacts on the power system. However, the resistive SFCL appears to be the more suitable to limit the fault current and to increase the transient stability of the power system in case of short-circuit.
\end{abstract}

Keywords: Inductive Superconducting Fault Current Limiter, Resistive Superconducting Fault Current Limiter, Electrical Power System, Current Limitation, System Stability, Short-Circuit.

\section{Introduction}

The addition of generation capacity due to the elevation of electrical demand tends to increase the destructive overcurrent when a fault occurs in the power system, taxing the capabilities of installed equipment, such as circuit breakers. Faults can be caused by equipment failures, severe weather, accidents or even acts of willful destruction [1]. Such faults can damage major, expensive components and, if not cleared quickly, can lead to lengthy and costly

${ }^{*}$ Corresponding author

Email addresses: gaetan.didier@univ-lorraine.fr (G. Didier), charles-henri.Bonnard@polymtl.ca (C. H. Bonnard), thierry.lubin@univ-lorraine.fr (T. Lubin), jean.leveque@univ-lorraine.fr (J. Lévêque) 
outages. Superconducting materials with high critical temperature (i.e. $>77 \mathrm{~K}$, which is the temperature of liquid nitrogen) is one of the most promising technologies for this application.

Superconducting fault current limiters use one of the fundamental properties of superconductors $[2,3]$. The operation of a SFCL is based on the sudden transition from the superconducting state to the normal state by exceeding the critical current $I_{c}$ of the material [4]. This transition from the superconducting to the normal state take a very short time, so fast that we are abble to limit the first current peak to a threshold value which not exceed three to five times the rated current. These limiters use the variation of resistivity during the transition. Indeed, the resistivity of these materials is very high, much more than a classical conductor, both for $\mathrm{BiSCCO}$ and for $\mathrm{YBaCuO}$ at $77 \mathrm{~K}$. The limiter is placed in series with a circuit breaker. During the fault, the current increases up to reach the threshold of transition from superconducting wire. This transition from the superconducting element to normal state causes the development of resistance in the case of a resistive SFCL while it modifies the inductance value in the case of an inductive SFCL. The time between threshold crossing and the limitation is small (a few milli-seconds). The circuit breaker isolates the line as soon as possible after the beginning of the limitation. The main advantages of the SFCL are:

- Very low impedance during normal operation. The current limiter is "invisible" in this mode. Some transients such as the inrush current during the energization of a transformer should not inadvertently cause a state change for the SFCL.

- High impedance system during short-circuit. The limiter must perform its function in the case of massive short circuit but also in the case of low short-circuit fault.

- Very good dynamic. The transition from superconducting to resistive or inductive state should be as fast as possible to be sure that the first peak of the fault current is properly limited (typically within millisecond)

In this way, such apparatus could reduce the prospective short-circuit current in the range of $20 \%$ to $50 \%$. In addition to the short-circuit current limitation, several studies have shown that the use of SFCL in an Electrical Power Grid (EPG) allows to improve the transient stability of generators and consequently the global stability of the network [5][6]. Several papers deal with the study of iSFCL $[7,8]$ or rSFCL $[9,10,11,12,13]$ or the both $[14,15]$ either in term of current limitation, or transient stability study. Kozak and al in [16] compare the two types of SFCLs experimentally but concentrate only on the decrease of the fault current. At this day, no comparative theoretical studies in term of current limitation and power system transient stability have been done between these two types of SFCLs on the same electrical power system.

In this paper, the iSFCL and rSFCL are compared in terms of current limitation and power system stability on a single machine infinite bus (SMIB). The influence on the current limitation is studied with the analysis of the short circuit current in transmission lines and at the feeder of the generator for the three states of the power system (prefault, fault and post-fault). These values take into account the impedance introduces by the SFCL in the system when a short-circuit occurs. The transient stability study is done by the analysis of the CCA and CCT of the power system in 
case of fault. The first one is evaluated by using the equal area criterion while the second is evaluated by the resolution of swing equations of the power system.

In addition with the comparative study, we observe the influence of the SFCL location in the power system. The use of SFCL in a power system allows to decrease the fault current and increase the power system stability [5]. Results of the both studies (comparison between iSFCL and rSFCL and between two possible locations) give us a good way to select the optimal SFCL and its optimal location in case of fault.

\section{Integration of SFCL in a simplified power system - Theoretical study}

To evaluate the effectiveness of the SFCL in a power system in terms of current limitation and power system stability, the equivalent circuit given at Fig. 1 is modeled using Matlab/Simulink software [15]. Parameters of this power system are given in Appendix A with a base power equal to 100 MVA. In this system, the generated power at the power plant is transferred by two transmission lines to an infinite bus represented by its voltage $\underline{V}=V \angle 0$. To evaluate the effect of the two types of SFCL on the power system, a short-circuit is created at point A (in the middle of the transmission line $L_{2}$ ). To complete the study of the two SFCLs, two possible locations of the limiter are analyzed, at the transformer feeder (TF) (position 1 at Fig. 1) or at the line feeder (LF) (position 2 at Fig. 1). The SFCL at the TF could allows to decrease the transformer series impedance which is a good way for voltage control. Such opportunity could be interesting in the case of a new power plant or substation. In fact, if the fault current is reduced by the SFCL, the design of protection devices is positively impacted that induce a reduction of the cost of the global protection system. In the case where the power system exists, this location allows to increase the transmitted power without the need to replace the existing protection apparatus. If the SFCL is placed at the LF, in addition with the limitation of the fault current, the voltage drop is limited on the others transmission lines ( $L_{1}$ in our case). This voltage quality improvement is very important specifically, for sensitive loads.

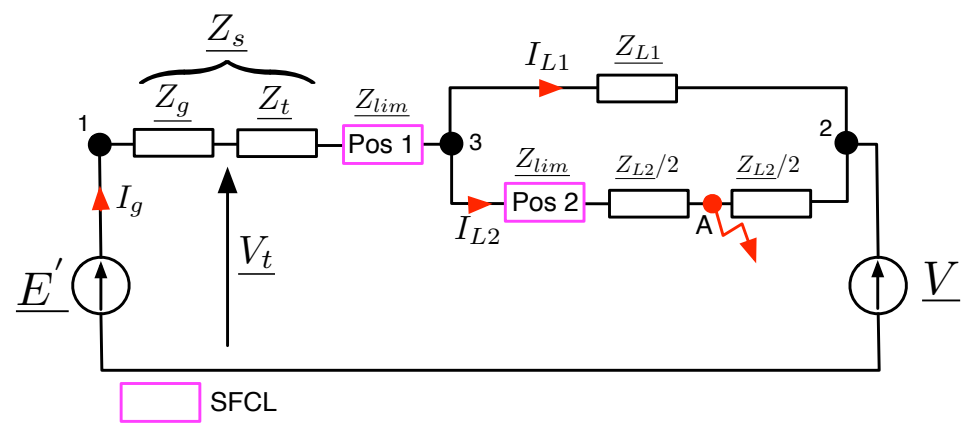

Figure 1: On-line diagram of the power system studied. 


\subsection{Analysis of current limitation with SFCL}

As a first step, we analyse the effect of the position of the SFCL on the current limitation if a fault occurs at point A. For each positions of the SFCL, a time domain approach is used to obtain the evolution of the three currents of the Single Machine Infinite Bus (SMIB) [17]. For simulations, a short-circuit is introduced at point A at $t_{f}=0.2 \mathrm{~s}$ and cleared at $t_{c}=0.3 \mathrm{~s}$. The SFCL impedance (resistive or inductive) is introduced at position 1 or 2 at $t_{\text {lim }}=0.201 \mathrm{~s}$ to take into account the time needed to change state from superconducting to resistive or inductive state. The value of short-circuit currents in the SMIB for different values of the SFCL impedance is analyzed and commented. We can give an orientation about the optimal location of the SFCL and for the most effective current limitation in an EPG.

\subsubsection{SFCL placed at the transformer feeder (position 1)}

To consider the iSFCL or rSFCL in the SMIB, the impedance $\underline{Z_{l i m}}$ is considered equal to $R_{l i m}$ for the resistive type and ${ }_{J} X_{l i m}$ for the inductive type. The line and generator currents are evaluated for different values of the SFCL impedance $\underline{Z_{\text {lim }}}$. In our case, these impedance are taken in a range from 0 to 0.4 p.u. for the both SFCLs (see Appendix A for the base impedance) that correspond to the existing value of SFCL for the transmission lines used in simulation. A SFCL impedance equal to 0 p.u. correspond to the study without SFCL in the SMIB. In nominal steady state, current values are equal to 0.5 p.u for $I_{L 1}$ and $I_{L 2}$ and 1 p.u. for $I_{g}$.

The evolution of the first peak current (during the fault) of $I_{L 1}, I_{L 2}$ and $I_{g}$ in accordance with the SFCL impedance is given at Figs. 2(a), 2(b) and 3 for a short-circuit at point A and the both types of SFCL placed at the transformer feeder. We can see that the first peak in the line current $I_{L 2}$ without SFCL (SFCL impedance at 0 ) is equal to 6.3 p.u. which correspond to 12 times the rated current. The analysis of Fig. 2(b) shows that the first peak of the fault current $I_{L 2}$ decreases with the presence of the both SFCLs (iSFCL or rSFCL). However, for this SFCL location, the resistive type allows a greater reduction of the line current than the inductive type for all SFCL impedances studied. For example, if we take the SFCL impedance at 0.3 p.u., the limited line current $I_{L 2}$ with the use of a rSFCL is more than $14 \%$ greater than with iSFCL.

However, the current level in the fault transmission line can not be the only parameter used to select the type of SFCL that can be used in an EPG. The evolution of all currents in the power system have to be analyzed. Consequently, if we have a look at line current $I_{L 1}$ (Fig. 2(a)), we can see an increase of its value according to the rSFCL and iSFCL impedance. Fig. 2(a) shows a major increase of $I_{L 1}$, in comparison with the fault current level without SFCL, if a rSFCL is used instead of an iSFCL with the same SFCL impedance ( $40 \%$ for the rSFCL and $17 \%$ for the iSFCL for a SFCL impedance equal to 0.3 p.u.). First results show that if a fault occurs at point $A$, the line current where the SFCL is placed decreases while the line current in the parallel line increases that can induce a breaker trip of the parallel transmission line and a stop of the power transferred to the inline bus.

About generator current $I_{g}$ (Fig. 3), both SFCLs allow to reduce the fault current but the most effective is the resistive one. In fact, with impedance values in the range 0.2-0.4, the fault current is limited at around half the value without SFCL and can reduce the electrical and mechanical effects on generator. 


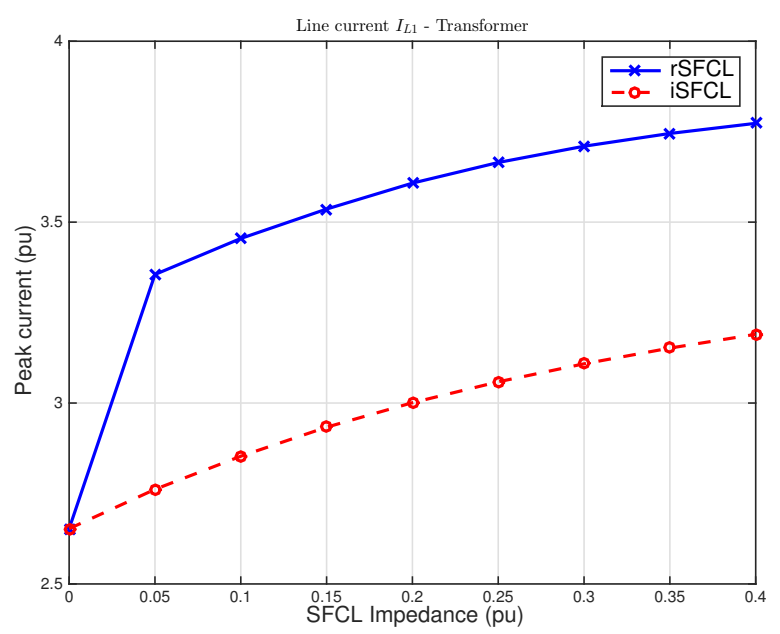

(a)

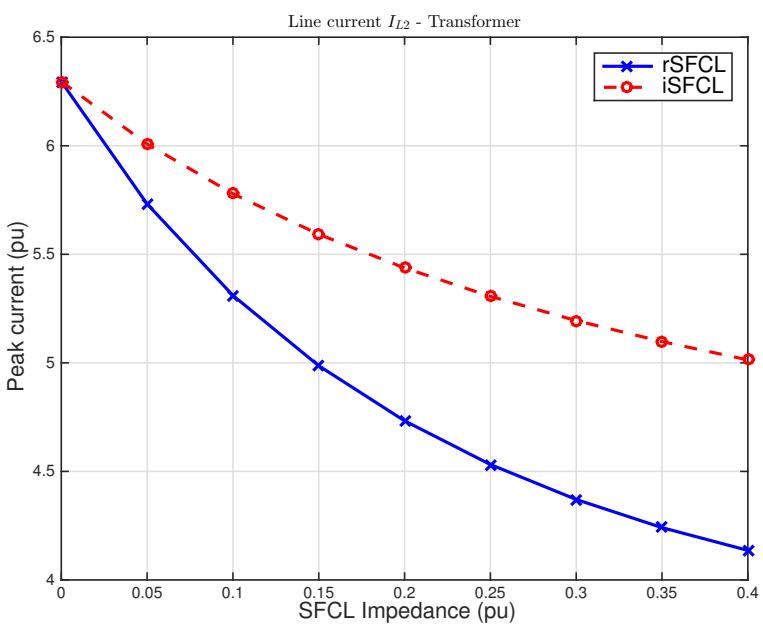

(b)

Figure 2: Line currents $I_{L 1}$ (a) and $I_{L 2}$ (b) for a resistive or inductive SFCL placed at TF.

\subsubsection{SFCL placed in the line feeder (position 2)}

With a SFCL placed at the line feeder, we made the same analysis than previously. For this position, differential equations of the power system have been solved and the evolution of currents $I_{L 1}, I_{L 2}$ and $I_{g}$ in accordance to the impedance value of the limiter are given at Figs. 4(a), 4(b) and 5 respectively. If a fault occurs in the transmission line $L_{2}$, the presence of a rSFCL at the line feeder decreases the first peak of fault current $I_{L 2}$ as we can see at Fig. 4(b). We can observe the same result with an iSFCL, but the reduction of the fault current magnitude is lower (2.96 p.u. for an iSFCL in comparison with 2.04 p.u. for a rSFCL for the same 0.4 p.u. impedance).

About $I_{L 1}$, we can see at Fig. 4(a) a significant reduction of its value for the both types of SFCL. In fact, when a fault appears at point A with a SFCL placed at the line feeder, the current peak $I_{L 1}$ is reduced for the two types of SFCL. However, it is with rSFCL that offers the best results (0.44 p.u. for rSFCL in comparison with 1.47 p.u. for iSFCL for an impedance equal to 0.4 p.u.). About $I_{g}$ (Fig. 5), the SFCL located at line feeder allows to decrease generator (and transformer) fault current magnitude in case of fault. The rSFCL appears to be the most suitable but the difference between them in term of current limitation is not very significant (1.89 p.u. for the rSFCL and 2.13 p.u. for the iSFCL for $Z_{\text {lim }}=0.4$ p.u.). However, we can note a good reduction of the fault current magnitude $I_{g}$ with the presence of the SFCL at line feeder. In fact, for a 0.4 p.u. SFCL impedance, we obtain a reduction of $46 \%$ for the iSFCL and $52 \%$ for the rSFCL.

If we compare the two positions of the SFCL, the line feeder seems to be preferable for two reasons. Firstly, the line current magnitude $I_{L 1}$ does not increase as we can see at Figs. 2(a) and 4(a) in case of short-circuit. In fact, the line feeder position with an iSFCL allow to reduce all currents magnitudes in the electrical power system studied in the opposite to rSFCL. Secondly, we obtain a greater limitation of fault currents if the SFCL is placed at line feeder 


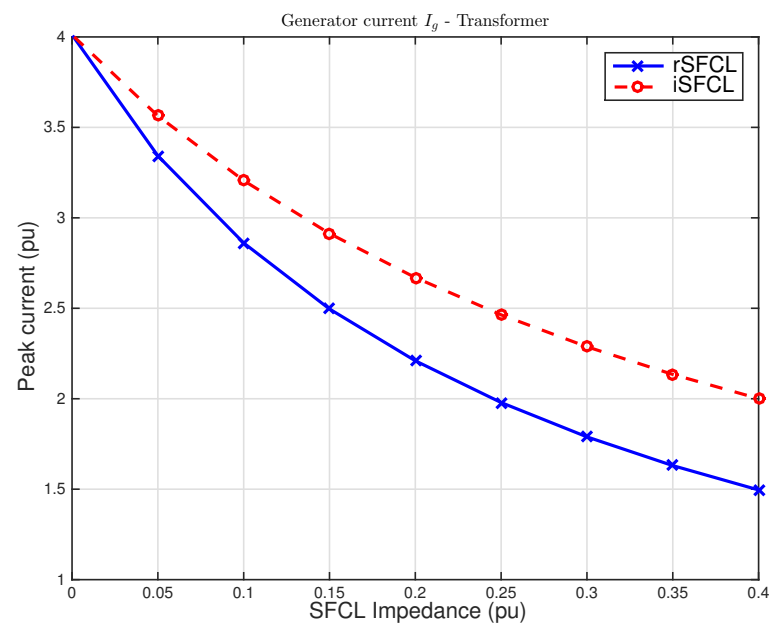

Figure 3: Generator current $I_{g}$ for a resistive or inductive SFCL placed at the transformer feeder.

(50\% better for a rSFCL and $40 \%$ for an iSFCL in comparison with TF position). If we limit the study to the fault current levels for a SFCL positioned at the line feeder or transformer feeder, the resistive type is better than the inductive type. In fact, the presence of an rSFCL, when a fault appears at point A, allows to reduce the three currents if the line feeder position is selected as explained previously.

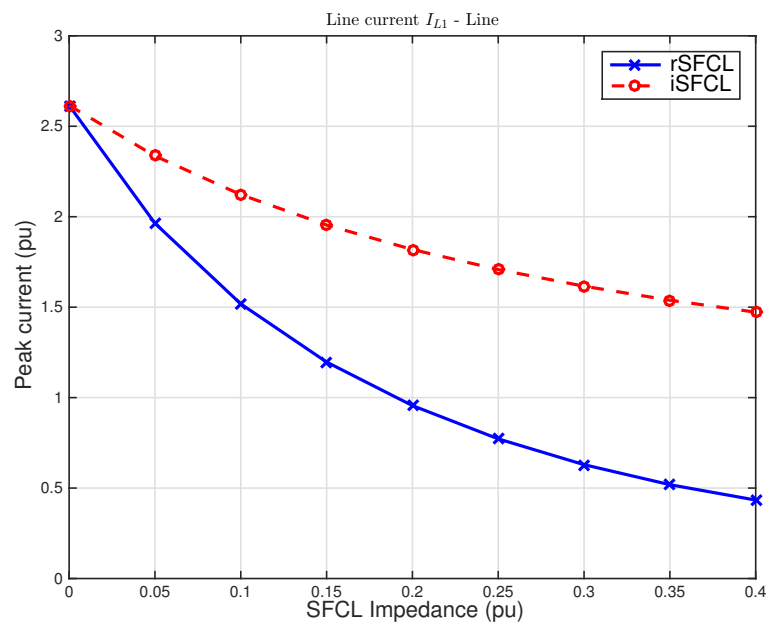

(a)

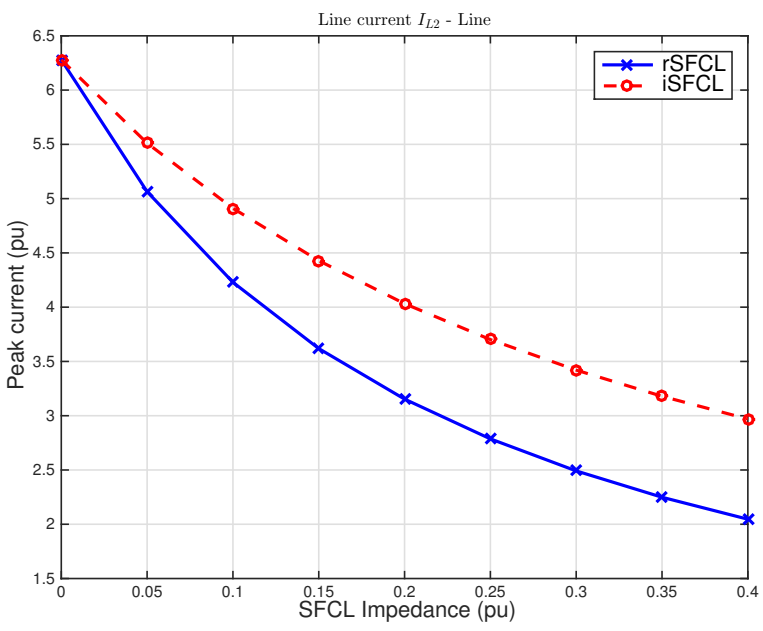

(b)

Figure 4: Line currents $I_{L 1}$ (a) and $I_{L 2}$ (b) for a resistive or inductive SFCL placed at LF.

We showed that the rSFCL is better to use than the iSFCL at line feeder. These results have been calculated by considering transmission lines of the power system purely inductive. To analyse the influence of the transmission lines parameters on fault currents level, additional simulations with various $X_{L} / R_{L} \operatorname{ratios}\left(Z_{L}=R_{L}+\jmath X_{L}\right)$ were 


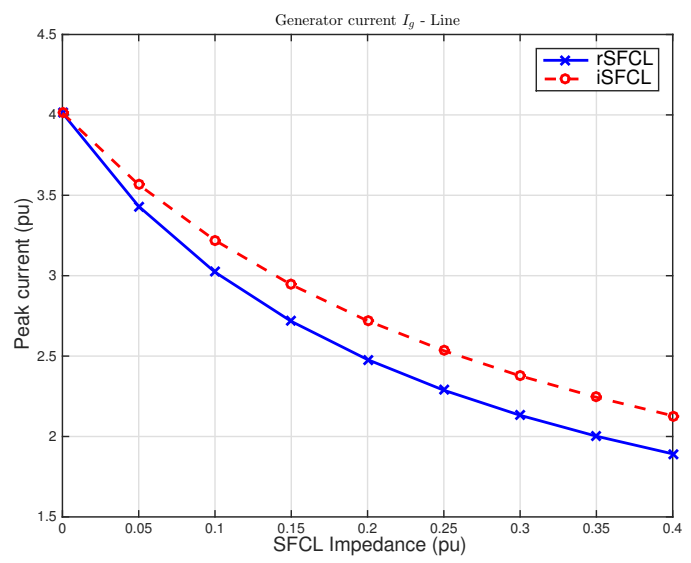

Figure 5: Generator current $I_{g}$ for a resistive or inductive SFCL placed at the line feeder.

done for a fault at position $A$. We give, for the two positions of the SFCL (TF and LF), the evolution of the ratio $I_{L 2}(r S F C L) / I_{L 2}(i S F C L)$ for a $X_{L} / R_{L}$ line equal to 5, 10 and 15 at Figs. 6.

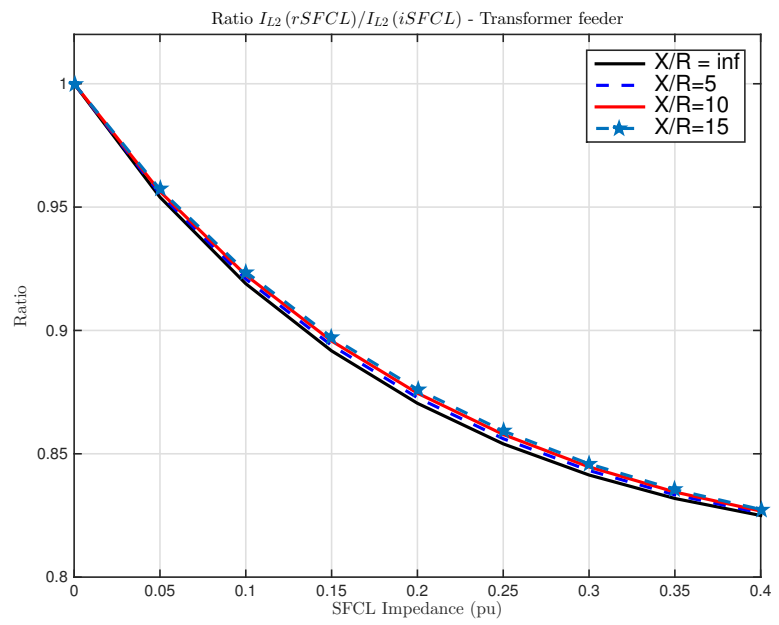

(a)

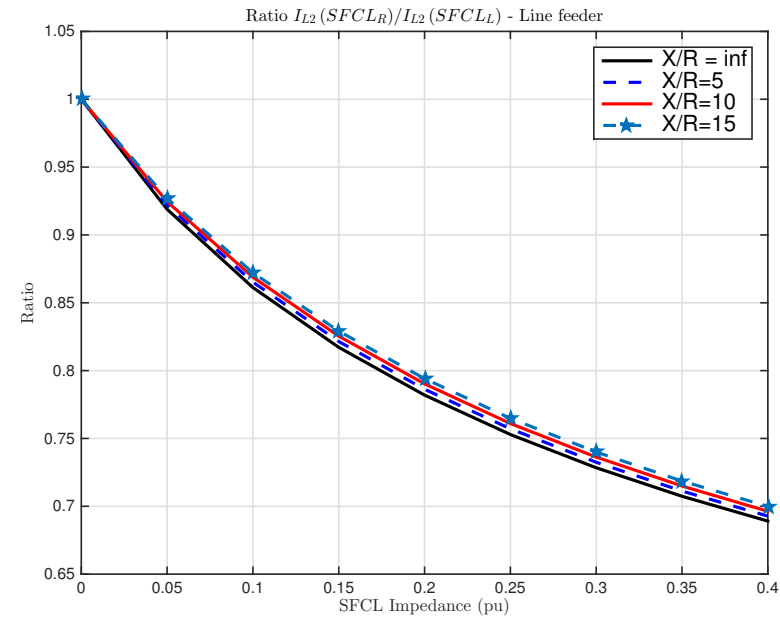

(b)

Figure 6: $I_{L 2}(r S F C L) / I_{L 2}(i S F C L)$ for different $X_{L} / R_{L}$ line ratios - (a) SFCL at TF and (b) SFCL at LF.

As we can see, all points are under 1 that means that the rSFCL remains the most suitable to limit the fault current in the faulty line $L_{2}$ for the both positions studied (transformer feeder and line feeder). In the same way, various positions of the fault in the transmission line $L_{2}$ were studied (in the middle of the line $(1 / 2-1 / 2)$ at the $3 / 10$ of the line length (3/10-7/10) and at the $7 / 10$ of the line length (7/10-3/10)). The rSFCL gives better results than iSFCL in term of current limitation as we can see at Fig. 7. Moreover, we can note that the rSFCL allows a more significant limitation in comparison with the iSFCL if the fault appears at the beginning of the line (3/10-7/10 in our case). 


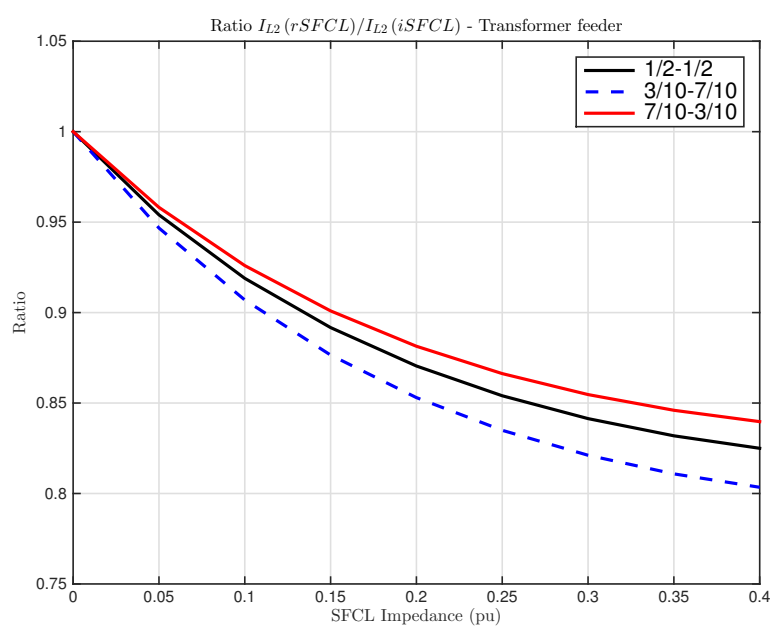

(a)

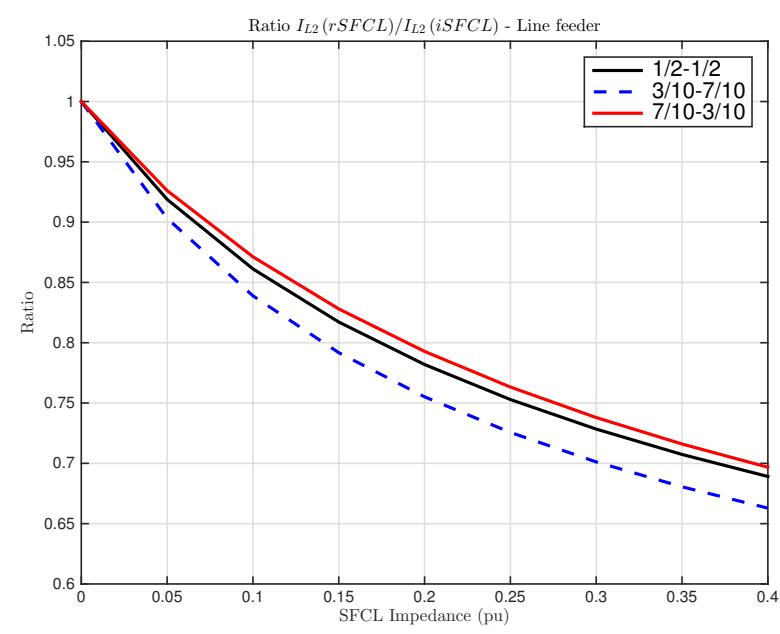

(b)

Figure 7: $I_{L 2}(r S F C L) / I_{L 2}(i S F C L)$ for different fault positions - (a) SFCL at TF and (b) SFCL at LF.

\subsection{Analysis of the critical clearing angle and critical clearing time with SFCL}

In this section, results presented concern the critical clearing angle (CCA) and the critical clearing time (CCT) for the two SFCLs studied at the two previous locations (with different values of the SFCL impedance). To evaluate the CCA of the SMIB in case of fault without and with SFCL, swing equation given at Eq. 1 and electrical power angle equation given at Eq. 2 are used. The power $P_{e}$ corresponds to the electrical power transmitted between nodes 1 and 2 of the SMIB as $P_{m}$ represents the mechanical power received by the generator [1].

$$
\begin{gathered}
\frac{d^{2} \delta}{d t^{2}}=\frac{\omega}{2 H}\left(P_{m}-P_{e}-D \frac{d \delta}{d t}\right) \\
P_{e}=\left|\underline{E^{\prime}}\right|^{2} \Re\left\{\underline{Y_{11}}\right\}+\left|\underline{E^{\prime}}\right| \underline{V}\left|\underline{Y_{12}}\right| \cos \left(\delta-\varphi_{12}\right)
\end{gathered}
$$

In these equations, $\delta, \omega, H, D, \underline{Y}, \varphi_{12}$ are respectively the angular difference of induced voltages at nodes 1 and 2, angular speed, inertia constant, damping coefficient, admittance transfer matrix between nodes 1 and 2 and angle of the complex term $\underline{Y_{12}}$. To evaluate the value of the CCA, the equal area criterion is applied to the proposed SMIB by considering a constant mechanical power $P_{m}$ during the fault. For each state of the system (pre-fault, fault and post-fault), the transfer matrix $\underline{Y}$ is evaluated to find the value of the CCA. The evaluation of the CCA is made by considering the opening of the faulty line $L_{2}$ after the fault (post-fault state). The CCA is evaluated with different values of the SFCL impedance (from 0 p.u., which correspond to no SFCL included in the grid, to 0.04 p.u.) for the two positions studied in this paper. 


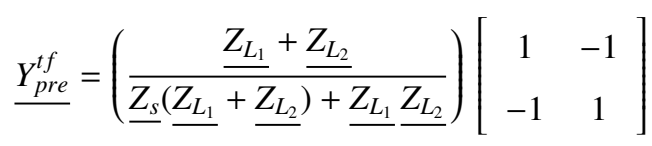

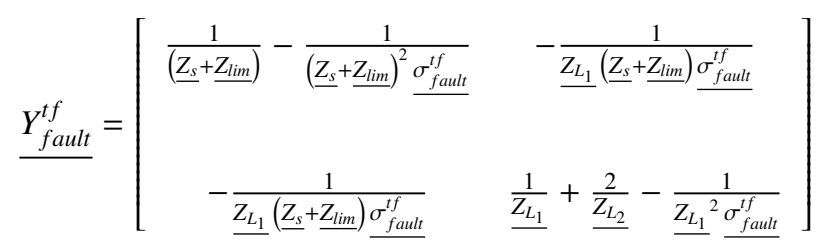

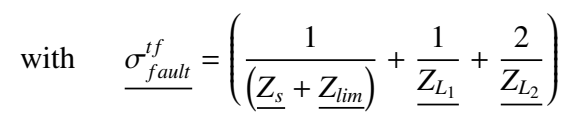

$$
\begin{aligned}
& \underline{Y_{\text {post }}^{t f}}=\left(\underline{\underline{Z_{s}}+\underline{Z_{\mathrm{L}_{1}}}}\right)\left[\begin{array}{cc}
1 & -1 \\
-1 & 1
\end{array}\right]
\end{aligned}
$$

\subsubsection{SFCL placed at the transformer feeder (position 1)}

For this position, the transfer matrices $\underline{Y}^{t f}$ for the three states of the SMIB (pre-fault, fault, post-fault) are given by Eqs. 5, 6 and 7 by considering parameters given in Appendix A. In the transfer matrix $Y_{p r e}^{t f}$, corresponding to the pre-fault condition, the term $Y_{\text {pre }_{11}}^{t f}$ presents only an imaginary part that give us a power $P_{e}$ exclusively dependent on the angle $\delta$ (see Eq. 2). If we plot the evolution of this electrical power according to angle $\delta$, we obtain the curve given at Fig. 8 (for the pre-fault state).

In case of fault condition, the power system is modified and the transfer matrix $Y_{\text {fault }}^{t f}$, given at Eq. 6, shows that if we place a rSFCL at the TF, the electrical power $P_{e}$ has a constant element due to the term $Y_{\text {fault }_{11}}^{t f}$. This constant element allows to absorb a part of the generated power during the fault. We propose, at Fig. 8, the evolution of the electrical power $P_{e}=f(\delta)$ for the three states of the power system (pre-fault, fault and post-fault) and for the two types of SFCL (we arbitrarily chose a SFCL impedance equal to 0.03 p.u for the study). Without fault, the power system operates with an initial angle $\delta$ equal to $0.456 \mathrm{rd}$. In case of fault with no SFCL, the equal area criterion gives us a CCA equal to $1.6 \mathrm{rd}$. This angle correspond to the equality between area ABCD and DEF. If an iSFCL is placed at the transformer feeder, we can see that the $P_{e}=f(\delta)$ curve is below that the curve without SFCL. This position increases the area between ABCD and consequently decreases the value of the CCA at $1.52 \mathrm{rd}$. Concerning the rSFCL, it places $P_{e}=f(\delta)$ above the curve with no SFCL. The consequence on the power system stability is that the area between $\mathrm{ABCD}$, according to mechanical power $P_{m}$, is reduced, that increase the CCA at $2.01 \mathrm{rd}$.

The evaluation of the CCA in case of SFCL placed at TF for various SFCL impedances values is given at Fig. 9(a). We can see that the presence of the rSFCL at the TF increases the CCA of the power system for all SFCL impedances studied. Concerning the inductive one, the CCA decreases with the increase of the SFCL impedance.

The CCT is evaluated by solving Eq. 1 with the use of the runge kutta of order 4 method [18]. The evolution of CCT in accordance with SFCL impedance for the TF position is given at Fig. 9(b) for a damping coefficient $D$ equal 


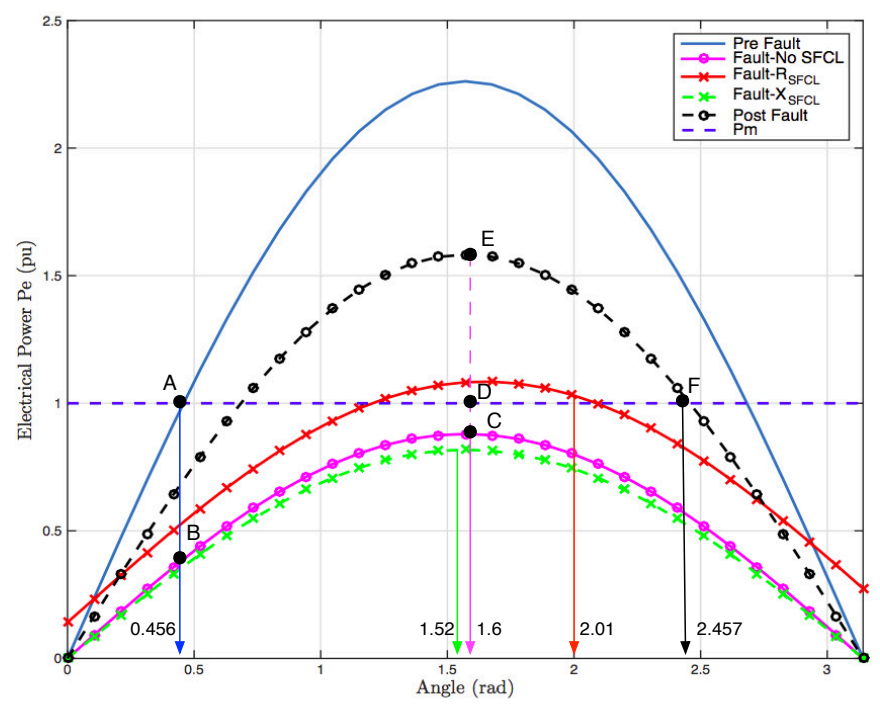

Figure 8: Equal area criterion of the SMIB for a fault at point A without SFCL and with a rSFCL or an iSFCL placed at the transformer feeder (pre-fault, fault and post-fault states), $\underline{\left|Z_{\text {lim }}\right|}=0.03$ p.u.

to 0.005 p.u. If the rSFCL is used, we obtain an increase of the CCT equal to $0.215 \mathrm{~s}$ in comparison with no SFCL (for $\left|\underline{Z_{\text {lim }} \mid}\right|$ equal to 0.03 p.u.). Concerning the use of iSFCL placed at the transformer feeder, the same conclusion as CCA can be done. In fact, we can see that the CCT decreases with the increase of the SFCL impedance. For example, the CCT decreases to $0.085 \mathrm{~s}$ for a SFCL impedance $Z_{\text {lim }}$ equal to 0.03 p.u. (compared to result with no SFCL). If the SFCL is located at the TF, it is better to use the rSFCL if the goal is to increase the CCA (or CCT) of the power system.

\subsubsection{SFCL placed at the line feeder (position 2)}

In this case, matrix $\underline{Y_{p r e}^{l f}}$ is identical to matrix $\underline{Y_{p r e}^{t f}}$ given at Eq. 5 and matrix $\underline{Y_{p o s t}^{l f}}$ is equal to matrix $\underline{Y_{p o s t}^{t f}}$ (the SFCL is short-circuited to recover its superconducting state). Without fault, the term $\underline{Y_{p r e} e_{11}}$ presents an imaginary part as explain in section 2.2.1. Evolutions of the electrical power $P_{e}$ according to angle $\delta$ for the three states of the power system and for the both SFCLs studied are given at Fig. 10. For this specific location, we can see that $P_{e}=f(\delta)$ curves for the both SFCLs are above the curve without SFCL. The area defines by ABCD decreases, so the CCA value increases in case of fault for the both SFCLs. However, it is for the rSFCL that we obtain best results. In fact, angle $\delta$ takes a value equal to $1.67 \mathrm{rd}$ for the iSFCL and $1.82 \mathrm{rd}$ for the $\mathrm{rSFCL}$, if we consider the same impedance $\underline{Z_{\text {lim }}}$. If we generalize the study for different values of $\underline{Z_{\text {lim }}}$, we obtain results given at Fig. 11(a). As for the TF location, we can see that the use of a rSFCL placed at the LF position allows to increase the CCA value in comparison with the iSFCL for the same impedance $Z_{\text {lim }}$. 


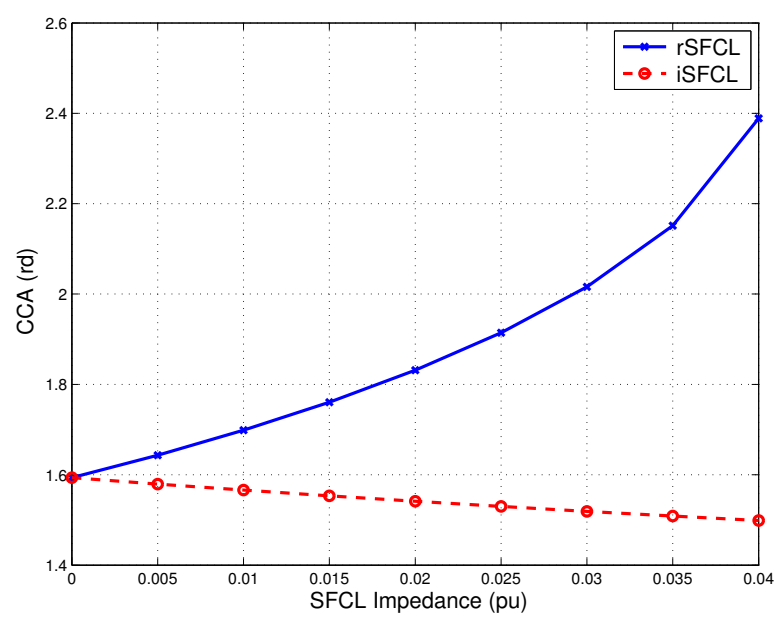

(a)

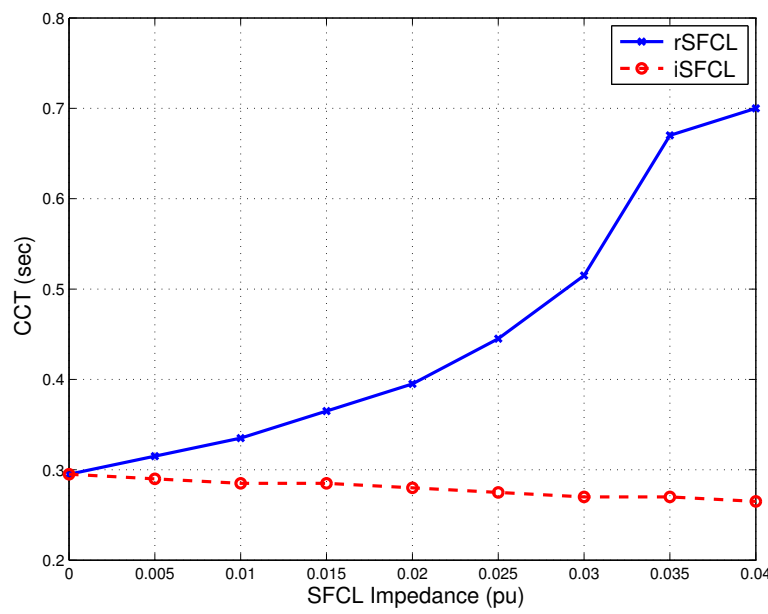

(b)

Figure 9: CCA (a) and CCT (b) for a resistive or inductive SFCL placed at TF.

$$
\begin{aligned}
& \underline{Y_{\text {fault }}^{l f}}=\left[\begin{array}{cc}
\frac{1}{\underline{Z_{s}}}-\frac{1}{\underline{Z_{s}^{2}} \underline{\sigma_{\text {fault }}^{l f}}} & -\frac{1}{\underline{Z_{s}} \underline{Z_{L_{1}}} \underline{\sigma_{\text {fault }}^{l f}}} \\
-\frac{1}{\underline{Z_{s}} \underline{Z_{L_{1}}} \underline{\sigma_{\text {fault }}^{l f}}} & \frac{1}{Z_{L_{1}}}+\frac{2}{\underline{Z_{L_{2}}}}-\frac{1}{\underline{Z_{L_{1}}^{2}} \underline{\sigma_{\text {fault }}^{l f}}}
\end{array}\right] \\
& \text { with } \quad \sigma_{\text {fault }}^{l f}=\left(\frac{1}{Z_{s}}+\frac{1}{Z_{L_{1}}}+\frac{1}{\underline{Z_{L_{2}}} / 2+\underline{Z_{\text {lim }}}}\right)
\end{aligned}
$$

If we make a comparison between TF and LF position, we can see that the CCA presents its optimal value when the SFCL is considered to be resistive and placed at the TF. In fact, for the same fault position (point A) and the same value of the SFCL impedance (for example 0.035 p.u.), we obtain the best CCA (equal to 2.18 rd) for a rSFCL (in comparison with $1.508 \mathrm{rd}$ for the iSFCL placed at TF, $1.876 \mathrm{rd}$ for the rSFCL at LF and $1.684 \mathrm{rd}$ for the iSFCL at LF).

Concerning the CCT, we give its evolution in accordance with SFCL impedance for the same damping coefficient $D$ used previously ( $D=0.005$ p.u) at Fig. 11(b). If the line feeder is selected, we can see that the rSFCL gives better results compared to iSFCL even if both increase the CCT for a specific SFCL impedance. If we take $\left|Z_{\text {lim }}\right|$ equal to 0.03 p.u, the rSFCL increases the CCT to $0.115 \mathrm{sec}$ in comparison to $0.03 \mathrm{sec}$ for the iSFCL. As the TF location, if the LF location is chosen, the rSFCL is more suitable concerning the increase of CCA and CCT.

In a same way that the current limitation study, the influence of $X_{L} / R_{L}$ line ratio and of fault position in transmission line $L_{2}$ on the CCA was studied. We give at Figs. 12(a) and 12(b) the evolution of the ratio $C C A(r S F C L) / C C A(i S F C L)$ in accordance to the SFCL impedance. Analysis shows that for all $X_{L} / R_{L}$ ratios, the rSFCL remains the best SFCL 


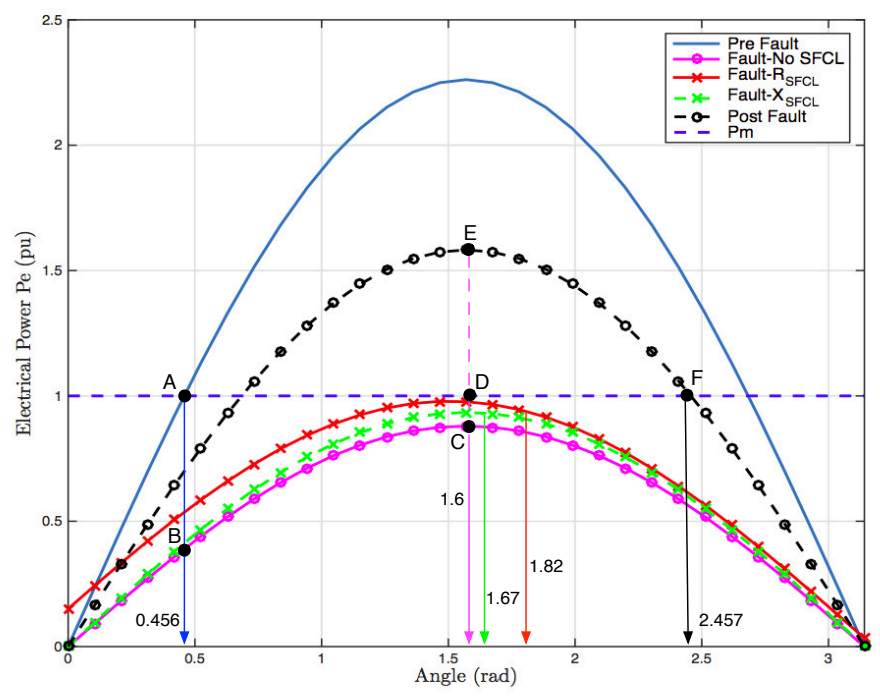

Figure 10: Equal area criterion of the SMIB for a fault at point A without SFCL and with a resistive or an inductive SFCL placed at the line feeder (pre-fault, fault and post-fault states), $\left|Z_{\text {lim }}\right|=0.03$ p.u.

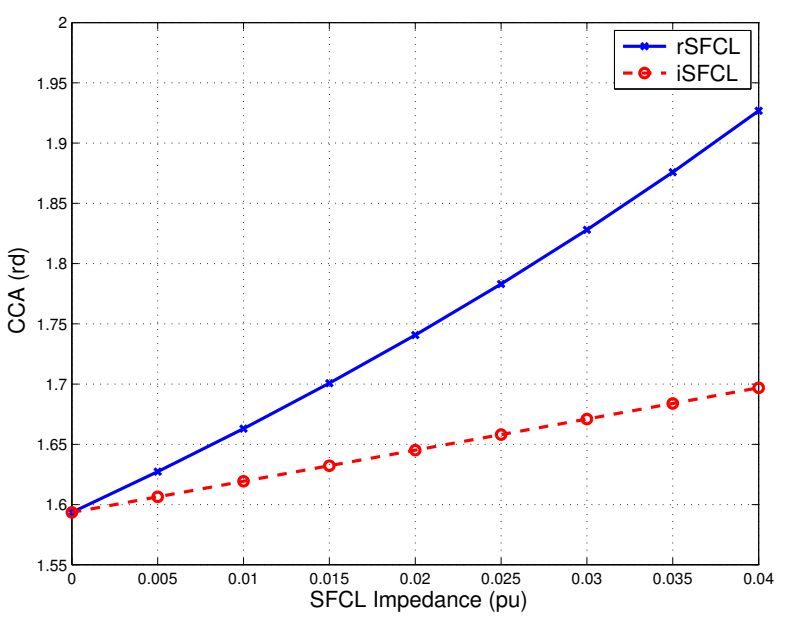

(a)

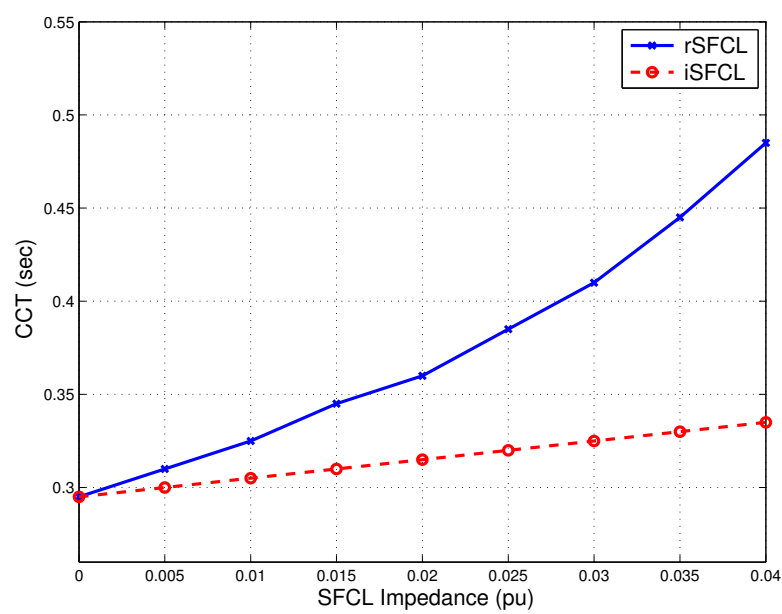

(b)

Figure 11: CCA (a) and CCT (b) for a resistive or inductive SFCL placed at LF.

for the power system transient stability. We can note that the difference induces by $X_{L} / R_{L}$ on the CCAs ratio is non significant. In fact, if we take a SFCL impedance equal to 0.04 p.u., the CCA ratio is in the range [1.44 - 1.51]. Moreover, it is important to note that the CCA value increases with the SFCL impedance if a rSFCL is used (1.5 if the SFCL is placed at TF and 1.13 if it is placed at LF). 


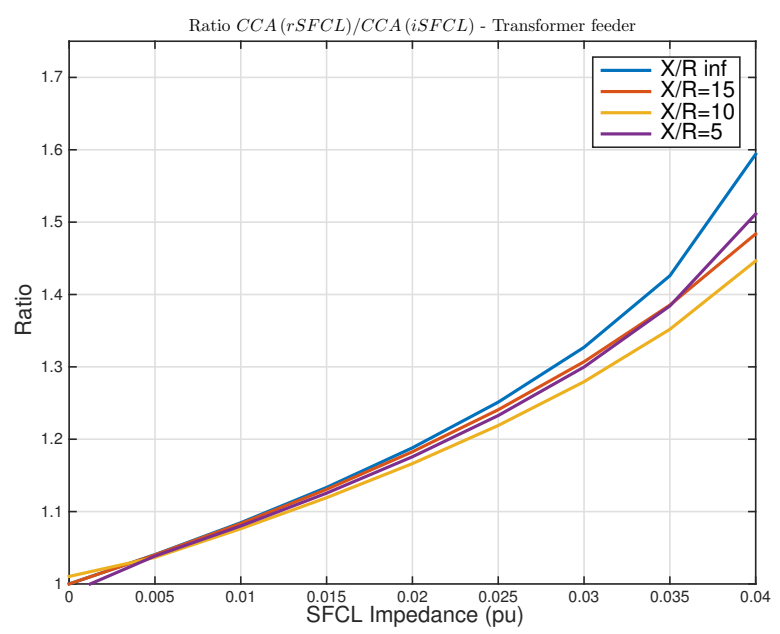

(a)

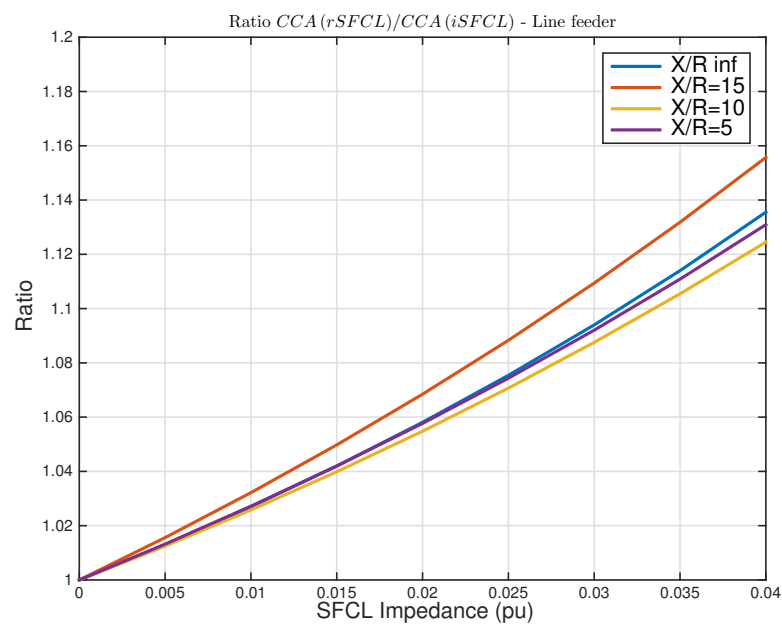

(b)

Figure 12: $C C A(r S F C L) / C C A(i S F C L)$ for different $X_{L} / R_{L}$ line ratios - (a) SFCL at TF and (b) SFCL at LF.)

Concerning the influence of fault positions on the CCA with rSFCL and iSFCL, Figs. 13(a) and 13(b) give the evolution of CCA ratio $C C A(r S F C L) / C C A(i S F C L)$ depending on SFCL impedance. The evolution of the CCA ratio shows that the rSFCL is the most suitable to increase the transient stability of the EPG. In fact, the evolution of the CCA ratio is above 1 for all SFCL impedances studied and for the two positions selected. Indeed, it is for the TF position that we obtain the optimal CCA ratio (1.58 for a $1 / 2-1 / 2$ fault).

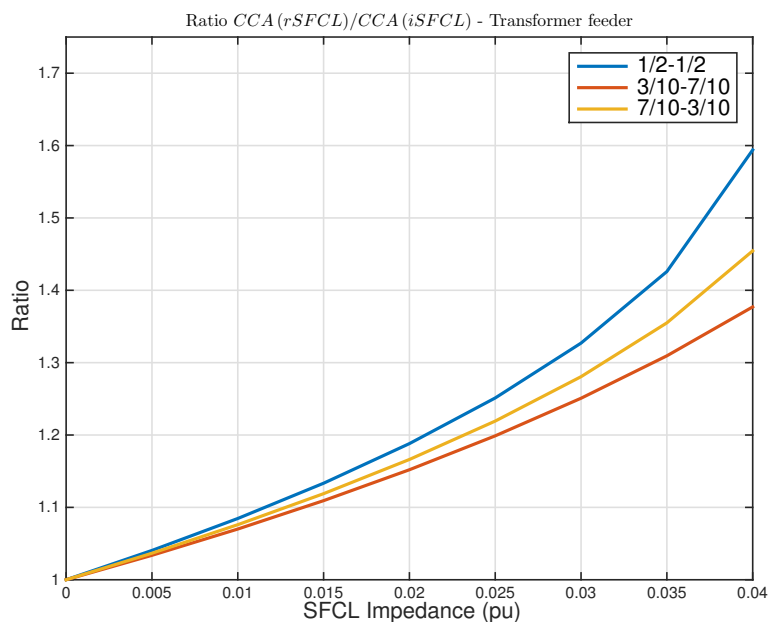

(a)

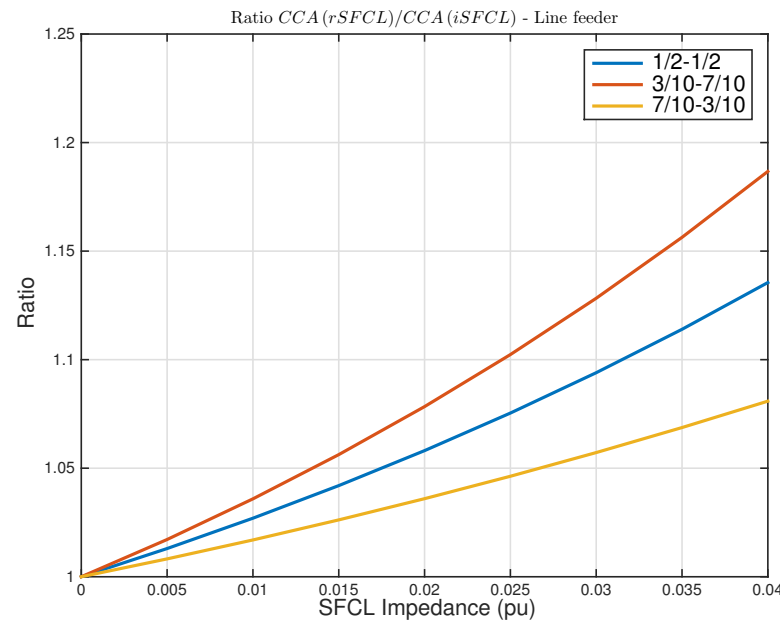

(b)

Figure 13: $C C A(r S F C L) / C C A(i S F C L)$ for different fault positions - (a) SFCL at TF and (b) SFCL at LF.)

We specify that the influence of $X_{L} / R_{L}$ ratio and fault position on the CCT gives the same conclusions as the 
analysis of CCA. The rSFCL allows to increase the transient stability of the EPG more significantly than the iSFCL for a given position and a given SFCL impedance.

\section{Conclusion}

This paper presents a qualitative analysis to compare two types of SFCL (inductive and resistive) in an electrical power grid. Results show that the two types of SFCL have a positive impact on the power system for current limitation and transient stability for a selected optimal location. In fact, an iSFCL placed at TF for example, decreases the CCT and CCA of the power system in case of fault. The proposed analysis shows the superiority of the resistive SFCL in the two domains that are fault current limitation and power system transient stability. However, to obtain the optimal behaviour of the power system in case of fault, the rSFCL has to be placed at LF to avoid the increase of the first peak current in the parallel line $L_{1}$. Moreover, the reduction of fault current $I_{L 2}$ is better if the rSFCL located at LF (2.05 p.u. at LF and 4.14 p.u. at TF for a SFCL impedance equal to 0.4 p.u.). In conclusion, the LF with a rSFCL appears to be a good solution to limit the fault currents in electrical power grid. Such observation shows that it is important to develop tools to quantify the impact of SFCL at a specific location with a specific impedance.

Concerning results obtain for the transient stability study, the rSFCL appears to be the most suitable to increase the CCA and the CCT of the EPG studied. The presence of the term $\left|\underline{E^{\prime}}\right|^{2} \mathfrak{R}\left\{\underline{\left.Y_{11}\right\}}\right.$ in the electrical power transmitted between node 1 and node 2 can explain the obtained results. In fact, the presence of the rSFCL at TF or LF induces additional DC component in the power $P_{e}$ during the fault. Consequently, the curve $P_{e}=(\delta)$ for the rSFCL is above the curve with the iSFCL for a specific SFCL impedance. This position increases the area DEF (Figs 8 or 10) that give a CCA (and a CCT) more important that for an iSFCL. In case of short-circuit, the rSFCL can dissipate a part of the excessive accelerating generator power, increasing the stability-limit of the system and then enlarging the stability-region after the fault. The placement of the rSFCL at TF appears to be the optimal way to enhance power transient stability. In fact, the gain obtained in this case is $25 \%$ better for the CCA and $45 \%$ for the CCT (for a SFCL impedance equal to 0.04 p.u.).

A particular attention has been paid on the influence of fault positions and transmission lines parameters in the comparison between rSFCL and iSFCL. For the power system studied, the $X_{L} / R_{L}$ ratio or the fault position do not modify significantly results previously obtained, even in terms of current limitation and power transient stability. The optimal combination between SFCL type and its placement in the power system is not an easy task. Results presented previously give an orientation on the influence of the SFCL type associated to its position in terms of fault current limitation and power transient stability study. Moreover, it is important to note that the presented analysis is focused on non-salient poles generators, and thus results may present small divergences in the case of salient poles generators. Additional analysis must be made to optimize these choices in a much larger power system. However, the proposed method allows to select a good orientation about these different points. 


\section{Appendix A. Power system parameters}

Generator : $90 \mathrm{MVA}, 22 \mathrm{kV}, \underline{Z_{g_{p u}}}={ }_{j} 0.2$

Transformer : $100 \mathrm{MVA}, 22 / 110 \mathrm{kV}, \underline{Z_{t_{p u}}}={ }_{j} 0.064$

Trans. Lines : $110 \mathrm{kV}, \underline{Z_{L 1_{p u}}}=\underline{Z_{L 2 p u}}={ }_{j} 0.397$

Base power : 100 MVA

Base voltage $=22 \mathrm{kV}$ (Generator), $110 \mathrm{kV}$ (Trans. lines)

Base impedance $=4.84 \Omega$ (Generator), $121 \Omega$ (Trans. lines)

\section{References}

[1] W. D. Stevenson, Elements of Power System Analysis, Mc Graw Hill International Editions, 1982.

[2] A. Morandi, State of the art of superconducting fault current limiters and their application to the electric power system, Physica C: Superconductivity 484 (2013) 242-247.

[3] L. Ye, A. Campbell, Behavior investigations of superconducting fault current limiters in power systems, IEEE Transactions on Applied Superconductivity 16 (2) (2006) 662-665. doi:10.1109/TASC.2006.870526.

[4] M. Noe, M. Steurer, High-temperature superconductor fault current limiters: concepts, applications, and development status, Superconductor Science and Technology 20 (3) (2007) R15.

[5] G. Didier, J. Leveque, A. Rezzoug, A novel approach to determine the optimal location of SFCL in electric power grid to improve power system stability, IEEE Transactions on Power Systems 28 (2) (2013) 978-984. doi:10.1109/TPWRS.2012.2224386.

[6] L. Ye, L. Lin, K.-P. Juengst, Application studies of superconducting fault current limiters in electric power systems, IEEE Transactions on Applied Superconductivity 12 (1) (2002) 900-903. doi:10.1109/TASC.2002.1018545.

[7] J. C. Aracil, J. Lopez-Roldan, J. C. Coetzee, F. Darmann, T. Tang, Analysis of electromagnetic forces in high voltage superconducting fault current limiters with saturated core, Int. Journal of Electrical Power and Energy Systems 43 (2012) 1087-1093.

[8] J. Kozak, T. Janowski, S. Kozak, G. Wojtasiewicz, B. Glowacki, Tests results and analysis of inductive superconducting fault current limiter with bi-2223 tube and hts tape as secondary winding, Journal of Physics 43 (2006) 946-949.

[9] H.-I. Du, Evaluation on current-limiting performance with the fault type of the 3-phase resistive type sfcls, IEEE Transactions on Applied Superconductivity 23 (3) (2013) 5603304-5603304. doi:10.1109/TASC.2013.2246757.

[10] A. Kudymow, S. Elschner, O. Maeder, W. Goldacker, Optimization of $2 \mathrm{~g}$ ybco wires for resistive fault current limiters, IEEE Transactions on Applied Superconductivity 21 (3) (2011) 1311-1314. doi:10.1109/TASC.2010.2086410.

[11] L. Ye, A. Campbell, Case study of HTS resistive superconducting fault current limiter in electrical distribution systems, Electric Power Systems Research 77 (2007) 534-539.

[12] P. J. Costa Branco, M. E. Almeida, J. A. Dente, Proposal for an rms thermoelectric model for a resistive-type superconducting fault current limiter (sfcl), Electric Power Systems Research 80 (2010) 1229-1239.

[13] S. Alaraifi, M. El Moursi, H. Zeineldin, Optimal allocation of hts-fcl for power system security and stability enhancement, Power Systems, IEEE Transactions on 28 (4) (2013) 4701-4711.

[14] A. Morandi, M. Bocchi, M. Fabbri, L. Martini, F. Legrini, P. L. Ribani, Comparison of the performances of three different types of fault current limiter in the distribution network, Journal of Physics.

[15] M. M. Aly, E. A. Mohamed, Comparison between resistive and inductive superconducting fault current limiters for fault current limiting, Computer Engineering and Systems. 
[16] J. Kozak, M. Majka, S. Kozak, T. Janowski, Comparison of inductive and resistive sfcl, IEEE Transactions on Applied Superconductivity 23 (3) (2013) 5600604-5600604. doi:10.1109/TASC.2012.2231714.

[17] D. Cvoric, S. De Haan, B. Ferreira, Cb stress reduction and comparison of energy dissipation for two types of fcls, Transmission and Distribution Conference and Exposition.

[18] B. Keyvani, M. K. Zadeh, H. Lesani, Stability enhancement of multi-machine systems using adaptive reclosing of transmission lines, International Journal of Electrical Power and Energy Systems 62 (2014) 391 - 397. doi:http://dx.doi.org/10.1016/j.ijepes.2014.04.013.

URL http://www.sciencedirect.com/science/article/pii/S0142061514001963 